\title{
Militärische Flugmedizin, quo vadis?
}

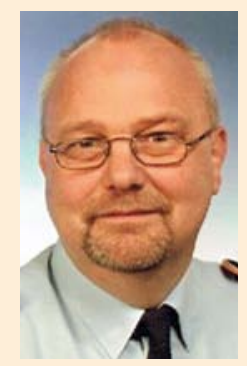

Liebe Kolleginnen und Kollegen,

als neues Vorstandsmitglied der DGLRM und im Editorial Board der FTR freue ich mich auf die neuen Aufgaben. Wenn Sie mehr über mich wissen möchten, dann können Sie in www.dglrm.de unter AG Militärische Flugmedizin mehr über mich finden. Die aktuellen, allumfassenden strukturellen Veränderungen (Stationierungskonzept 2011, Standortschließungen, Bündelungen der Aufgaben) und personellen Auswirkungen (Personalreduzierung, Aussetzung der Wehrplicht seit dem Sommer 2011, Nachwuchssorgen) in der Bundeswehr (Bundeswehrreform) aber auch die Einführung neuer hochkomplexer Waffensysteme („Kleiner, straffer, schlagkräftiger sowie langfristig bezahlbar und auf sinkende Geburtenzahlen vorbereitet.“) führte und führt zu bedeutsamen Veränderungen im fliegerärztlichen Dienst der Bundeswehr. Am 30.09.2013 wurden die Dienststellen „Generalarzt der Luftwaffe“ und das „Flugmedizinische Institut der Luftwaffe“ aufgelöst und am 01.10.2013 das Zentrum für Luft- und Raumfahrtmedizin der Luftwaffe geschaffen. Die 3 tragenden Säulen der militärischen Flugmedizin (Fachabteilungen) sind die Wissenschaft, die Begutachtung und das Kompetenzzentrum Fliegerärztlicher Dienst der Luftwaffe. Diese Reform schafft die Voraussetzungen für eine Kooperation mit dem DLR, eine sinnvolle und logische Bündelung der flugmedizinischen und raumfahrtmedizinischen Kompetenz und eine große Herausforderung für eine erfolgreiche zivil-militärische Kooperation. Dies bedingt einen Umzug der bisher in Fürstenfeldbruck und Manching beheimaten Anteile nach Köln-Wahn, aber erst nach Schaffung der notwendigen baulichen Voraussetzungen. Durch die personelle Reduzierung in Luftwaffe/Heer/Marine und die Einführung der neuen Waffensysteme EF-2000 Eurofighter, KHS TIGER, NH90 und A-400M mit weniger aber hochspezialisierten Luftfahrzeugführern und Besatzungsmitgliedern stehen der Mensch und seine Leistungsfähigkeit noch mehr im Mittelpunkt der militärischen Flugmedizin. Dieser Wandel ist in allen Partnerluftwaffen und fliegerärztlichen Diensten zu beobachten. So hielt der Surgeon General der USAF auf der 85. Jahrestagung der AsMA in San Diego am 11.05.2014 einen eindrucksvollen Festvortrag mit dem Titel „Human and Performance“. Neben den traditionellen und unveränderten Aufgaben der militärischen Flugmedizin wie Auswahl, Eignung, Tauglichkeit, Gesundheit, Fitness, taktischer und strategischer AIRMEDEVAC, Prävention und Schutz vor extremen Umweltbedingungen gewinnen nun die Erhaltung und Steigerung der Leistungsfähigkeit (Human Performance Enhancement) und die Gesundheitsfürsorge und Vorsorge an Bedeutung. Es gilt das Potenzial (Fähigkeiten) der Ressource Mensch zu erkennen, Fertigkeiten zu entwickeln, die Leistungs- und Durchhaltefähigkeit zu steigern und zu erhalten (mit legalen Mitteln und flugmedizinisch vertretbaren Mitteln) und den Piloten beziehungsweise das Besatzungsmitglied vor den Folgen extremer Belastungen zu schützen (Beschleunigungen, extremen Höhen, Kälte, Hitze, Kampfeinsatz, Verletzung, Verwundung, Erkrankung). Diese anspruchsvollen Aufgaben und Ziele meistern wir nur gemeinsam, als Fliegerarzt der Bundeswehr, als flugmedizinischer Sachverständiger, als Wissenschaftler. Sowohl die DGLRM als Fachgesellschaft als auch die FTR als Fachzeitschrift sind ein wichtiges Instrument für alle Flugmediziner sich zu präsentieren, sich auszutauschen und Kooperationen zu finden. Die aktuelle Ausgabe spiegelt die Vielfalt der flugmedizinischen Inhalte und Fragestellungen wieder. 